Check for updates

The BMJ

Cite this as: $B M J 2020 ; 371: \mathrm{m} 4307$ http://dx.doi.org/10.1136/bmj.m4307 Published: 05 November 2020

\section{Covid-19: NHS hospitals are urged to recruit more patients to Recovery trial to find what treatments work}

\section{Gareth lacobucci}

The head of the UK's Recovery trial has urged NHS hospitals to recruit more patients to the study in order to speed up the development of treatments for covid-19.

Recovery (randomised evaluation of covid-19 therapy) is the world's largest randomised trial of covid-19 treatments, recruiting around 150 patients a day.

Giving evidence to the Commons Science and Technology committee on 4 November, Peter Horby, the study's chief investigator and professor of emerging infectious diseases and global health at the University of Oxford, told MPs that UK development of treatments for covid-19 has been "mostly a story of success." Recovery was the trial that discovered that dexamethasone shows benefit in patients seriously ill with covid-19. ${ }^{1}$

Horby said improvements in the treatment and management of covid-19 patients appeared to have reduced mortality in the second wave, with figures suggesting that the fatality rate among hospitalised patients in England was currently around 15\%, compared with 30\% during the first wave.

But he said treatments that are still being tested could be rolled out more quickly if more patients were recruited to trials. "We're only recruiting about $10 \%$ of all patients admitted to hospitals," he told MPs. "We're talking about getting an answer on the effectiveness of convalescent plasma in six to eight weeks-if we doubled the recruitment, we'd get an answer in half the time. And that's true for the monoclonal antibodies and all the drugs that we're studying.

"Some NHS hospitals are recruiting 30-35\% of all covid-19 patients to the trial. We should be aiming for that kind of number. At the other end of the scale there are hospitals that have 200-300 patients with covid-19 and have recruited none."

Horby said that the study team had held talks with national NHS leaders and the National Institute for Health Research (NIHR) about ways to improve recruitment. "It comes down to resourcing research staff, empowering non-research staff to get involved in research, and to some extent performance management of some trusts," he said.

"This is a national effort. If we want to find more treatments like dexamethasone before Christmas, we need to get $10 \%$ recruitment up to $30 \%$ and then we'll find answers much quicker."

But Horby acknowledged that staff in hospitals are under "extreme pressure" to recruit not just to covid-19 trials but to other study areas too. "We have to be careful not to browbeat people on the ground," he said. "We need to talk to them and work with the NIHR and the NHS executive to support them to recruit patients." He also said it was important for patients and their relatives to be aware of the availability of the trials and the importance of them.

Horby said that the Recovery trial had been designed to be part of routine care, which he suggested could be an effective model for conducting future trials. "This is something that can be done by nurses and doctors on the wards. There's a lesson there for research in the NHS going forward.”

Mahase E. Covid-19: Low dose steroid cuts death in ventilated patients by one third, trial finds. BMJ2020;369:m2422. doi: 10.1136/bmi.m2422 pmid: 32546467 